\title{
Mitochondrial haplogroups modify the effect of black carbon on age-related cognitive impairment
}

\author{
Elena Colicino ${ }^{1 *}$, Melinda C Power ${ }^{1,2}$, David G Cox ${ }^{3,4}$, Marc G Weisskopf ${ }^{1,2}$, Lifang Hou ${ }^{5}$, Stacy E Alexeeff ${ }^{1}$, \\ Marco Sanchez-Guerra ${ }^{1}$, Pantel Vokonas ${ }^{6}$, Avron Spiro $\mathrm{II}^{6}$, Joel Schwartz ${ }^{1,2}$ and Andrea A Baccarelli, ${ }^{1,2}$
}

\begin{abstract}
Background: Traffic-related air pollution has been linked with impaired cognition in older adults, possibly due to effects of oxidative stress on the brain. Mitochondria are the main source of cellular oxidation. Haplogroups in mitochondrial DNA (mtDNA) mark individual differences in oxidative potential and are possible determinants of neurodegeneration. The aim of this study was to investigate whether mtDNA haplogroups determined differential susceptibility to cognitive effects of long-term exposure to black carbon (BC), a marker of traffic-related air pollution.

Methods: We investigated 582 older men ( $72 \pm 7$ years) in the VA Normative Aging Study cohort with $\leq 4$ visits per participant (1.8 in average) between 1995-2007. Low ( $\leq 25)$ Mini Mental State Examination (MMSE) was used to assess impaired cognition in multiple domains. We fitted repeated-measure logistic regression using validated-LUR BC estimated in the year before their first visit at the participant's address.

Results: Mitochondrial haplotyping identified nine haplogroups phylogenetically categorized in four clusters. BC showed larger effect on MMSE in Cluster 4 carriers, including I, W and X haplogroups, [OR $=2.7 ; 95 \% \mathrm{Cl}(1.3-5.6)]$, moderate effect in Cluster 1, including J and T haplogroups [OR $=1.6 ; 95 \% \mathrm{Cl}:(0.9-2.9)]$, and no effect in Cluster 2 ( $\mathrm{H}$ and $\mathrm{V}$ haplogroups) $[\mathrm{OR}=1.1 ; 95 \% \mathrm{Cl}$ : (0.8-1.5)] or Cluster 3 ( $\mathrm{K}$ and $\mathrm{U}$ haplogroups) $\mathrm{OOR}=1.0 ; 95 \% \mathrm{Cl}$ : (0.6-1.6)]. BC effect varied only moderately across the I, X, and W haplogroups or across the J and T haplogroups.

Conclusions: The association of $\mathrm{BC}$ with impaired cognition was worsened in carriers of phylogenetically-related mtDNA haplogroups in Cluster 4. No BC effects were detected in Cluster 2 and 3 carriers. MtDNA haplotypes may modify individual susceptibility to the particle cognitive effects.
\end{abstract}

Keywords: mtDNA haplogroups, Air pollution, Black carbon, Cognitive decline, Mini-mental state examination

\section{Background}

Recent epidemiologic investigations have linked agerelated loss of cognition to long-term exposure to particulate air pollution [1-3]. A form of particulate matter is Black Carbon (BC), which is often used as a marker of air pollution from traffic sources. BC exposure has been specifically associated with lower cognitive performance [3]. The pathways linking traffic-related air pollution to cognitive impairment have not yet been thoroughly clarified [4].

Mitochondria are membrane-enclosed organelles that perform metabolic reactions to create energy in the

\footnotetext{
* Correspondence: ecolicin@hsph.harvard.edu

'Department of Environmental Health, Harvard School of Public Health, 665

Huntington Ave, Boston, MA 02115, USA

Full list of author information is available at the end of the article
}

form of Adenosine-5'-triphosphate (ATP) through multienzyme complexes located in both the inner mitochondrial membrane and the mitochondrial matrix [5]. Mitochondrial dysfunction has been linked to human age-related and neurogenerative disorders, due at least in part to augmented mitochondrial reactive oxygen species production [6]. Because air pollution effects on cognition have been suggested to be mediated through generation of oxidative species [7], markers of mitochondrial dysfunction may help clarify the mechanisms activated by the exposure and identify individuals at higher risk of exposure-related cognitive impairment. Mitochondria have their own DNA (mtDNA) enclosed in a single circular chromosome that is inherited through the maternal lineage. This mtDNA codes for 13 essential polypeptide components of the mitochondrial respiratory chain, two ribosomal Ribonucleic 
acids (RNA)s, and 22 transfer RNAs. mtDNA has a higher mutation rate than nuclear DNA [8]. Consequently, a considerable number of mtDNA point mutations (singlenucleotide polymorphisms) have accumulated sequentially along maternal lineages [9]. A number of stable polymorphic sites have been identified in mtDNA coding regions that define related groups of mtDNA, called haplogroups [9]. The haplogroups may be further grouped into clusters according to their phylogenetic background [9]. Because of the relevance of mtDNA for energy production and oxidative stress generation, mtDNA haplogroups and clusters have been intensively investigated in relation to aging and neurodegerative diseases characterized or accompanied by cognitive impairment, including Alzheimer's disease [10], and frontal lobe disease [11].

In the present study, we haplotyped participants in a large prospective cohort of aging and categorized them according to clusters of mtDNA haplogroups. We hypothesized that mtDNA haplogroups modified the effects of $\mathrm{BC}$ on cognitive function.

\section{Methods}

Study sample

The US Department of Veterans Affairs (VA) Normative Aging Study (NAS) is an ongoing longitudinal cohort established in 1963, which included men who were 2180 years of age and free of known chronic medical conditions at entry $[12,13]$. Participants were subsequently invited to medical examinations every three to five years. Starting in 1993, all participants completed a battery of cognitive tests. We excluded participants who had experienced a stroke ( $3 \%$ of individuals), leaving a total of 582 individuals with complete BC, mtDNA haplogroup, and covariate data. The study was approved by the Institutional Review Boards (IRB)s of the participating institutions. Participants have provided written informed consent at each visit. Counts of eligible and non-eligible participants and reason for ineligibility are reported in Additional file 1: Table S1.

\section{Cognitive testing}

We considered the Mini-Mental State Examination (MMSE), which was administrated with consistency over the period 1995-2007 among the cohort participants. MMSE is a test of global cognition that assesses multiple cognitive areas, i.e., orientation, immediate and shortterm recall, attention and calculation, word finding, figure construction, reading and writing skills, and ability to follow a 3-step command. The range of scores is 0 to 30 . The MMSE is extensively validated and used in clinical practice and research, as well as in epidemiology, as a dementia screening [14]. In this study, the maximum MMSE score was 29, due to the exclusion of the MMSE question on the county of residence, which has limited political and administrative meaning in Massachusetts and thus not of diagnostic utility [14,15]. We included cognitive data from study visits performed since 1995 (i.e., the first year after a full year of $\mathrm{BC}$ exposure data could be estimated) through 2007. We defined as the baseline visit for each study participant the first cognitive assessment completed on or after July 1st, 1995 . Up to 4 cognitive testing were completed by the study participants and were all used in the statistical analysis. On average each participant completed 1.8 tests.

\section{Exposure assessment}

A validated spatio-temporal land-use regression model in the greater Boston, Massachusetts area was used to obtain daily estimates since 1994 of Black Carbon (BC) exposure at the residence of each participant $[3,16]$. The model develops daily BC predictions from daily BC estimates averaged from 83 monitoring sites. Included in the model were predictors based on meteorological conditions (e.g., wind speed), land use (e.g., traffic density), daily $\mathrm{BC}$ concentrations at a central monitor, and other descriptors (e.g., day of the week). The goodness of fit of the model, based on the training set, was high $\left(R^{2}=\right.$ 0.83 ) and the average correlation between predicted values and observed BC levels in four out-of-sample validation samples was moderate (average $R^{2}=0.59$ ). For the present study, addresses with predicted daily $\mathrm{BC}$ concentrations outside the range of the exposure measurements from the training set were excluded. We considered as a metric of long-term exposure the average of the 365 daily estimates at the participant's residential address before the date of the baseline cognitive assessment of each participant [3].

\section{Mitochondrial (mtDNA) haplogroups}

Genotyping was conducted on blood DNA using Taqman assays (Applied Biosystems, Foster City, CA) for all polymorphisms, with the exception of 8994 which was genotyped using Sequenom due to Taqman design failure. Primer and probe sequences, as well as cycling conditions are available upon request. Internal blinded quality control samples were $>99 \%$ concordant, and all samples yielded genotypes. Hardy-Weinberg equilibrium tests are not valid for mitochondrial SNPs, and therefore were not assessed. No heteroplasmy (detected as heterozygous samples) was observed. The mtDNA haplogroups found in the cohort were $\mathrm{H}, \mathrm{I}, \mathrm{J}, \mathrm{K}, \mathrm{T}, \mathrm{U}, \mathrm{V}, \mathrm{W}$, and $\mathrm{X}$, which are most common in populations of European ancestry (Figure 1). To limit multiple comparison testing, haplogroups were grouped into four clusters according to the phylogenetic evolutionary tree $[9,11]$. Because haplogroups are inherited through maternal lineages, matrilineal ethnicity was collected through questionnaire and categorized based on the selfreported maternal grandmother's ancestry. Four-hundred- 


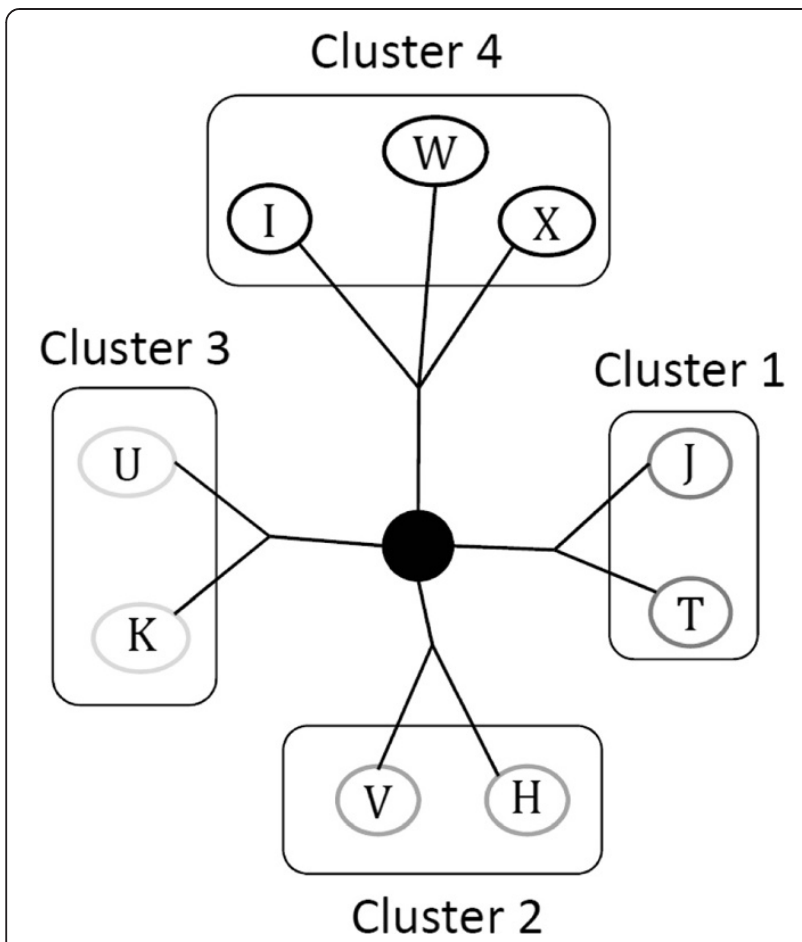

Figure 1 Position on a simplified phylogenetic evolutionary tree of the mtDNA haplogroups and clusters in NAS.

fifty-two individuals did not provide blood DNA for the haplogroup analysis and were therefore excluded from the study. The participants without haplogroup information were similar to the eligible participants for $\mathrm{BC}$ levels and MMSE scores (Additional file 1: Table S2).

\section{Statistical analysis}

A consistent proportion of participants (14\% of our observations) achieved the maximum score in the MMSE, thus revealing a ceiling effect for MMSE scores. Only 8\% of our observations exhibited scores $\leq 24$, i.e. the typical screening cut-off score used in research on dementia. Therefore we considered scores $\leq 25$ as low performance of MMSE (19\% of our observations) [3], created a dichotomous variable for low MMSE performance based on this cutoff, and used this in all models as the dependent variable.

We first evaluated the main effect of mtDNA clusters, as well as of $\mathrm{BC}$, on the odds of having a low MMSE score. We then evaluated whether the BC-cognition association was modified by clusters of mtDNA haplogroups. For clusters of mtDNA haplogroups that showed main effects or interactions, we planned on also evaluating individual haplogroups within each cluster. In all models, we used logistic regression with generalized estimating equations and empirical variance estimates to account for the repeated measures for each individual. All models were adjusted for potential confounders or predictors of cognitive function, including age at cognitive assessment (as a continuous variable) and several variables at baseline, i.e. education $(<12,12|-| 16,>16$ years $)$, matrilineal ethnicity (English speaking European ancestry, Mediterranean Europe, other European countries, other countries), first language (English/not English), computer experience (yes/no), smoking (current, former, never), body mass index (BMI) $(<25, \geq 25)$, physical activity $(<12$, $12 \mid-30, \geq 30$ metabolic equivalent hours (MET-hr) per week), alcohol intake ( $<2$ drinks/day, $\geq 2$ drinks/day), diabetes (yes/no), hypertension (yes/no), dark fish consumption (<once a week, zonce a week), percentage of the participant's census tract that is nonwhite, percentage of residential census tract adults with a college degree, indicator for first cognitive assessment (yes/no) to differentiate from the following assessments, and an indicator for whether the participant was a part-time resident of the greater Boston area (yes/no). Education, computer experience and dark fish consumption also served as proxies of SES and diet quality for each participant.

A sensitivity analysis was conducted adjusting for only age and both age and education at the baseline.

A change in cognitive function was also examined to complete the analysis. The dependent variable consisted in the difference between the MMSE score at the second, third and fourth visit and the first cognitive assessment. We used linear regression models with generalized estimating equations and empirical variance estimates. The potential confounders or predictors of the change of cognitive function included in the model were the same baseline variables described above, as well as age at baseline and the age difference between age at the visit and age at baseline.

Because of the log-linear relationship between $\mathrm{BC}$ and MMSE, as indicated by restricted cubic splines [3], a log-transformation of $\mathrm{BC}$ was used in the all analyses. We used SAS (version 9.2; SAS Institute Inc., Cary, NC) for all analyses.

\section{Results}

\section{Study participants characteristics}

The mean age of the study participants at baseline was 72 years (Standard Deviation (SD) 7; range, 53-97 years). Most men reported English as their first language (86\%) and alcohol consumption $<2$ drinks per day (75\%), were current or former smokers (70\%) and did not have diabetes mellitus (86\%). A minority were of normal adiposity (BMI $<25 ; 23 \%)$ or did not have hypertension (33\%) (Table 1). On the natural scale, 1-year average BC exposure estimates ranged between $0.03-1.77 \mu \mathrm{g} / \mathrm{m}^{3}$ $\left(\right.$ mean $\pm \mathrm{SD}, 0.6 \pm 0.3 \mu \mathrm{g} / \mathrm{m}^{3}$ ) and exhibited a right skewed distribution (details in $[3,16])$. We log-transformed BC (ln $(\mathrm{BC})$ ) and reported associations for a doubling in $\mathrm{BC}$ concentration on the natural scale, or approximately a 0.7 unit 
Table 1 Baseline characteristics of the Normative Aging Study (NAS) cohort ( $\mathbf{N}=582$ )

\begin{tabular}{lccc}
\hline Characteristics & N & $(\%)$ & $\begin{array}{c}\text { BC concentration }\left(\mu \mathrm{g} / \mathrm{m}^{3}\right) \\
\text { Mean } \pm \operatorname{StdDev}\end{array}$ \\
\hline
\end{tabular}

Age (Years)

50-59

60-69

70-79

80-89

90-99

Education (Years)

$<12$
$12|-| 16$
$>16$

First Language

$\begin{array}{lccc}\text { English } & 498 & (85.6) & 0.59 \pm 0.28 \\ \text { Not English/Bilingual } & 84 & (14.4) & 0.60 \pm 0.27 \\ \begin{array}{l}\text { Computer Experience } \\ \text { Yes }\end{array} & 242 & (41.6) & 0.56 \pm 0.26 \\ \text { No } & 340 & (58.4) & 0.61 \pm 0.29\end{array}$

Physical Activity (MET-hr/week)

$\begin{array}{llll}<12 & 324 & (55.7) & 0.59 \pm 0.29 \\ 12 \mid-30 & 160 & (27.5) & 0.61 \pm 0.29 \\ \geq 30 & 98 & (16.8) & 0.57 \pm 0.25\end{array}$

Alcohol (drinks/day)

$\begin{array}{lccc}<2 & 439 & (75.4) & 0.59 \pm 0.29 \\ \geq 2 & 143 & (24.6) & 0.58 \pm 0.25 \\ \text { Diabetes } & & & \\ \text { No } & 499 & (85.7) & 0.59 \pm 0.29 \\ \text { Yes } & 83 & (14.3) & 0.60 \pm 0.25\end{array}$

Consumed Dark Fish (times/week)

$\begin{array}{lrrr}<1 & 497 & (85.4) & 0.59 \pm 0.28 \\ \geq 1 & 85 & (14.6) & 0.57 \pm 0.28\end{array}$

Continued on the

next page

Continued from the

previous page

\section{Nonwhite (\% of census tract)}

$\begin{array}{lccc}<5 \% & 226 & (38.8) & 0.54 \pm 0.30 \\ 5 \% \mid-10 \% & 157 & (27) & 0.55 \pm 0.25 \\ \geq 10 \% & 199 & (34.2) & 0.68 \pm 0.26\end{array}$

$\geq \mathbf{2 5}$ years of age with at least a college degree (\% of census tract)

$\begin{array}{lccc}<30 \% & 192 & (33) & 0.64 \pm 0.30 \\ 30 \% \mid-50 \% & 230 & (39.5) & 0.56 \pm 0.28 \\ \geq 50 \% & 160 & (27.5) & 0.58 \pm 0.25\end{array}$

Table 1 Baseline characteristics of the Normative Aging Study (NAS) cohort $(\mathbf{N}=\mathbf{5 8 2}$ ) (Continued)

\begin{tabular}{lrrr}
\hline Smoking Status & & & \\
Never & 172 & $(29.6)$ & $0.57 \pm 0.25$ \\
Former & 34 & $(5.8)$ & $0.63 \pm 0.21$ \\
Current & 376 & $(64.6)$ & $0.60 \pm 0.30$ \\
BMI (Kg/m ${ }^{2}$ ) & & & \\
$<25$ & 136 & $(23.4)$ & $0.61 \pm 0.27$ \\
$\geq 25$ & 446 & $(76.6)$ & $0.58 \pm 0.28$ \\
$\begin{array}{l}\text { Hypertension } \\
\text { No }\end{array}$ & 190 & $(32.6)$ & $0.56 \pm 0.26$ \\
$\begin{array}{l}\text { Yes } \\
\text { Ethnicity }\end{array}$ & 392 & $(67.4)$ & $0.61 \pm 0.29$ \\
$\begin{array}{l}\text { English Speaking European } \\
\text { countries }\end{array}$ & 263 & $(45.2)$ & $0.61 \pm 0.30$ \\
$\begin{array}{l}\text { Mediterranean European } \\
\text { Countries }\end{array}$ & 141 & $(24.2)$ & $0.60 \pm 0.27$ \\
$\begin{array}{l}\text { Others European countries } \\
\text { Others }\end{array}$ & 113 & $(19.4)$ & $0.55 \pm 0.26$ \\
\hline
\end{tabular}

change in $\ln (\mathrm{BC})$ (details in [3]). The distribution of MMSE performance by mitochondrial clusters and haplogroups was reported in Additional file 1: Table S3. MMSE was associated with most of the potential confounders and predictors (Additional file 1: Table S4).

Frequency of mitochondrial haplogroups and clusters Cluster 1 included haplogroups $\mathrm{J}$ or $\mathrm{T}$ and was found in $19 \%$ of the participants. Cluster 2 included haplogroups $\mathrm{H}$ and $\mathrm{V}$ and was the most common among the participants (51\%), and was therefore taken as the reference category. Cluster 3 included haplogroups $\mathrm{K}$ and $\mathrm{U}$ and was found in $20 \%$ of participants. Cluster 4 included the most ancestral haplogroups I, W, and X and was found in $10 \%$ of the participants (Table 2). MtDNA haplogroups reported significant association with matrilineal ethnicity, BMI, first language, SES variables (education, dark fish consumption, computer experience), diseases (hypertension, diabetes) and life style variables (smoking status, alcohol intake) (Additional file 1: Table S4).

\section{BC effects on cognition and modification by mtDNA haplogroups}

In the entire study population, each doubling in $\mathrm{BC}$ on the natural scale was associated with 1.22 times higher odds (95\% Confidence Interval (CI): 0.95-1.56; Figure 2B; Additional file 1: Table S6) of low MMSE score adjusted for clinical and lifestyle factors. We examined the main effect of mtDNA haplogroup clusters on cognition by estimating the relative odds of low MMSE score relative to Cluster 2, taken as reference (Figure 2A; Additional 


\begin{tabular}{lcc}
$\begin{array}{l}\text { Table } 2 \text { Proportion of the mitochondrial clusters and } \\
\text { haplogroups in the Normative Aging Study (NAS) cohort } \\
\text { participants (N = 582) }\end{array}$ & N \\
\hline Haplotypes & $\mathbf{1 0 8}$ & \% \\
\hline Cluster $\mathbf{1}$ (J or T) & 50 & $\mathbf{1 9}$ \\
Haplogroup J & 58 & 9 \\
Haplogroup T & $\mathbf{2 9 6}$ & 10 \\
Cluster 2 (H or V) & 51 & $\mathbf{5 1}$ \\
Haplogroup H & 245 & 9 \\
Haplogroup V & $\mathbf{1 1 6}$ & 42 \\
Cluster 3 (K or U) & 55 & $\mathbf{2 0}$ \\
Haplogroup K & 61 & 10 \\
Haplogroup U & $\mathbf{6 2}$ & 10 \\
Cluster 4 (I, W or X) & 32 & $\mathbf{1 0}$ \\
Haplogroup I & 8 & 5 \\
Haplogroup W & 22 & 1 \\
Haplogroup X & & 4 \\
\hline
\end{tabular}

file 1: Table S5). None of the clusters showed an association with low MMSE score.

BC effects on MMSE varied by mtDNA haplogroup clusters (Figure 2B; Additional file 1: Table S6). BC effect was larger among Cluster 4 carriers (Odds Ratio (OR) = 2.70 for a doubling in BC concentration; 95\% CI: 1.305.59; OR for the interaction term $=2.46$; $95 \% \mathrm{CI}$ : 1.10 5.48). No effect of $\mathrm{BC}$ on MMSE was found in carriers of Cluster $2(\mathrm{OR}=1.10$ for a doubling in $\mathrm{BC}$ concentration; 95\% CI: 0.79-1.53; Cluster 2 was the reference category for the interaction term) or Cluster $3(\mathrm{OR}=0.97$ for a doubling in $\mathrm{BC}$ concentration; 95\% CI: 0.60-1.58; OR for the interaction $=0.89 ; 95 \% \mathrm{CI} 0.50-1.57$ ). A moderate borderline effect of $\mathrm{BC}$ on MMSE was found among Cluster 1 carriers $(\mathrm{OR}=1.62$ for a doubling in $\mathrm{BC}$ concentration; 95\% CI: 0.90-2.91; OR for the interaction $=1.48$; 95\% CI: 0.77-2.86).

To further characterize Cluster 4 and 1, we evaluated the individual mtDNA haplogroups included in the clusters taking as reference haplogroup $\mathrm{V}$, the most common haplogroup and part of Cluster 2 (Figure 2C; Additional file 1: Table S7). Estimates for the BC effects on low MMSE within each haplogroup had low precision, but they did not vary substantially across the three haplogroups in Cluster 4. In Cluster 1, we found larger BC effect in haplogroup J carriers $(\mathrm{OR}=3.04 ; 95 \% \mathrm{CI}: 1.18-7.86)$ and no effect in haplogroup T carriers $(\mathrm{OR}=0.88 ; 95 \% \mathrm{CI}$ : $0.39-2.00)$. We conducted sensitivity analyses using models adjusted for age only (Additional file 1: Table S8) or for age and education (Additional file 1: Table S9), but not for other covariates. Results from these models were consistent with those from the fully adjusted models.

To complete our analysis, we evaluated whether BC exposure was associated with differences in MMSE. Only a subset of the cohort participants had more than one cognitive assessment $(n=387)$ on which differences on MMSE could be calculated. In this analysis, clusters of mtDNA haplogroups did not show any main or modifier effects on the change of cognitive function (Additional file 1: Table S10-S11). A borderline effect of BC on change of cognition was found among haplogroup I carriers and haplogroup W carriers (Additional file 1: Table S12).

\section{Discussion}

In the present investigation, we found that clusters of mitochondrial haplogroups modified the association between long-term exposure to traffic air pollution - defined as the 1-year average of $\mathrm{BC}$ levels before the baseline assessment - and cognition in a cohort of older men. We showed that the $\mathrm{BC}$ effect on low performance in MMSE scores was limited to Cluster 4 carriers, and possibly Cluster 1 carriers. No association between BC and low cognition was found among carriers of Cluster 2 and 3. To our knowledge, this is the first study to investigate and identify mitochondrial haplogroups and their clusters as modifiers of effects of environmental exposures on cognitive aging.

Due to unique population histories, mtDNA haplogroups vary substantially in different areas of the world and reflect the history of the female lineage through human migrations [9]. According to the mtDNA evolutionary tree, Cluster 4 is composed by the oldest mtDNA haplogroups I, X and W [17]. Only few studies have evaluated the functional differences between mitochondrial haplogroups. Haplogroups J has been associated with increased L-strand transcription and mtDNA copy number [18]. Macrohaplogroup N, ND3 and ATP6 may affect complex I activity, membrane potential, and $\mathrm{Ca}^{11}$ regulation [19].

Air pollution exposure has been recently linked with increased levels of mtDNA damage, as reflected in increased mtDNA copy number [20] and reactive species of oxygen (ROS) generation [21,22]. ROS have been implicated in inflammatory cytokine production, suggesting a connection between oxidative stress and inflammatory processes [23]. MtDNA haplogroups may also affect the coupling of the respiratory chain [24], which can result in increased endogenous ROS in mitochondria [25]. Recent work has demonstrated that mtDNA haplogroups modify the relationship of traffic-related air pollution exposures with systemic biomarkers of inflammation [26], suggesting a role of mitochondrial haplogroups on systemic inflammation. The impact of these mechanisms on cognitive function or in local inflammation in the central nervous system has not yet been characterized. However Cluster 4 has been associated with frontotemporal lobar degeneration in a previous study in Finland [11]. Within both Cluster 4 and Cluster 1, the non- 

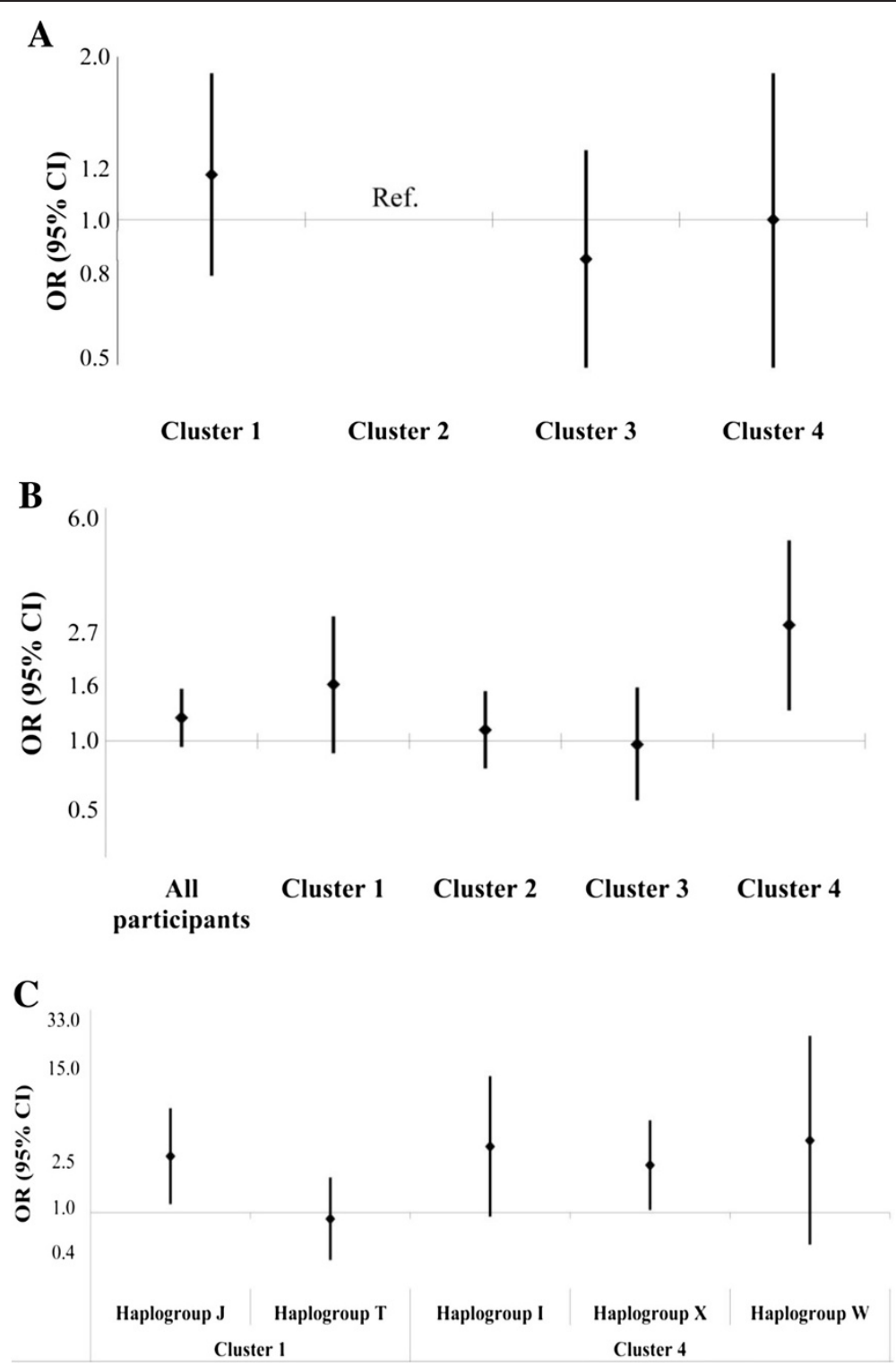

Figure 2 Main effect and effect modification of clusters and mitochondrial haplogroups on cognitive function. The figure shows the main effect of mtDNA haplogroups clusters on the low MMSE (Panel A); effect for doubling in BC concentration on the natural scale on the low MMSE score overall and by mitochondrial clusters (the most common cluster, i.e. Cluster 2, was taken as reference) (Panel B); and effect for doubling in BC concentration on the natural scale on the low MMSE score by individual haplogroups (the most common haplogroup, i.e. haplogroup V, was taken as reference) (Panel C).

synonymous/synonymous rate in mtDNA encoded genes has been shown to be higher than in the remaining European mtDNA haplogroup clusters [27]. The relative excess of nonsynonymous mutations in Clusters 4 and 1 has been suggested to play key roles in the risk of developing frontotemporal lobar degeneration [11]. In view of these findings, our results suggest that mtDNA clusters may be involved in determining the degree of damage in the central nervous system caused by environmental pollutants. MtDNA haplogroups might modulate exposureinduced inflammation and oxidative stress responses, which are both critical to determine loss of cognitive function throughout aging.

We recognize several limitations to our investigation. Our findings are based on a cohort of older men and may be generalized only to populations with similar age and gender characteristics. Additional studies are warranted to confirm our results among women and other ethnic group. Due to low proportions of most mtDNA haplogroups, we conducted our primary analysis on phylogenetic clusters. Larger studies are warranted to evaluate the effects of individual haplogroups within 
each cluster. The data used in the present study differ from our previous work in the NAS [3] in that we restricted the sample to study participants with mtDNA haplogroups and ethnicity information. Due to this restriction, we observed a less robust main effect of $\mathrm{BC}$ on cognitive function, possibly due, at least in part, to lower statistical power. We used geospatial models to estimate ambient $\mathrm{BC}$ and represent personal exposures of trafficrelated pollution. Most error involved in evaluating air pollution exposures are of Berkson's type. Simulation studies have shown this exposure misclassification is highly unlikely to bias away from the null and may rather lead to underestimation of air pollution effects [28]. Since BC concentration levels are spatially heterogeneous due to the numerous local (mobile) sources, measurement error in our BC exposure metric would likely reduce the true association. Thus, it is unlikely that this error would affect our conclusions.

\section{Conclusions}

Our results indicate that mtDNA haplogroups may modify the effects of traffic particles on cognitive impairments in this population of elderly men. If confirmed, these results may provide a novel approach to determine susceptibility to the toxic effects of traffic pollution, as measured by $\mathrm{BC}$, and contribute to growing understanding of the roles of mitochondria in the effects of environmental stressors.

\section{Additional file}

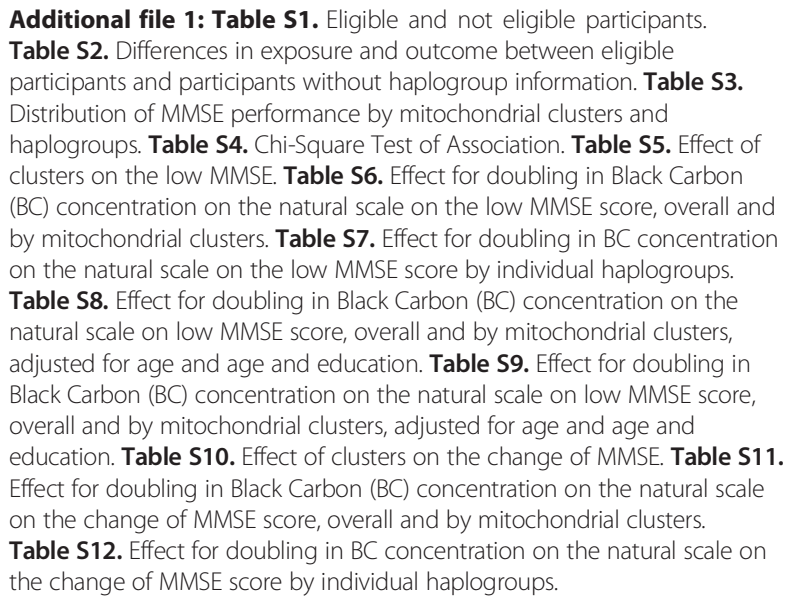

\section{Abbreviations}

ATP: Adenosine-5'-triphosphate; BC: Black carbon; BMl: Body mass index; Cl: Confidence interval; IRB: Institutional review board; LUR: Land use regression model; MET-hr: Metabolic equivalent hours; MMSE: Mini-mental state examination; mtDNA: Mitochondrial DNA; NAS: Normative Aging Study; OR: Odds ratio; RNA: Ribonucleic acid; SD: Standard deviation; VA: US Department of Veterans Affairs.

\section{Competing interests}

The authors declare that they have no competing interests.

\section{Authors' contributions}

EC performed the statistical analysis and drafted the manuscript. MCP contributed to perform the statistical analysis. DGC and MSG participated in data generation. MGW participated in developing the study concept and provided assistance in the interpretation of cognitive data. LH and SEA participated in developing the study concept and provided advice in performing the statistical analysis. PV initiated the study cohort and has conducted its follow-up. AS implemented cognitive testing in the cohort. JS developed exposure assessment strategies, directed the use of exposure data, and supervised statistical analysis. $A B$ developed the study concept, contributed to the interpretation of the data and helped to draft the manuscript. All authors read and approved the final manuscript.

\section{Authors' information}

EC: Postdoctoral Research Fellow in Biostatistics. Department of Environmental Health, Harvard School of Public Health, Boston, MA, USA. MCP: PhD. Department of Environmental Health and Department of Epidemiology, Harvard School of Public Health, Boston, MA, USA. DGC: BS MS PhD. Research Associate. Cancer Research Center of Lyon, Lyon, France. MGW: Associate Professor of Environmental and Occupational Epidemiology. Department of environmental Health and Department of Epidemiology, Harvard School of Public Health, Boston, MA, USA. LH: Associate Professor in Preventive Medicine. Department of Preventive Medicine Northwestern University Feinberg School of Medicine, Chicago, IL, USA. SEA: PhD Department of Biostatistics, Harvard University, Boston, MA, USA. MSG: Postdoctoral Research Fellow. Department of Environmental Health, Harvard School of Public Health, Boston, MA, USA. PV: MD. VA Boston Healthcare System University Schools of Public Health and Medicine, Boston, MA, USA. AS: Research Professor. VA Boston Healthcare System University Schools of Public Health and Medicine, Boston, MA, USA. JS: Professor of Environmental Epidemiology. Department of environmental Health and Department of Epidemiology, Harvard School of Public Health, Boston, MA, USA. AAB: Mark and Catherine Winkler Associate Professor of Environmental Epigenetics Department of Environmental Health and Department of Epidemiology, Harvard School of Public Health, Boston, MA, USA.

\section{Acknowledgements}

EC is supported by a grant from the National Institute of Environmental Health Sciences (NIEHS) (R01ES021733). Other support comes from NIEHS grants ES015172, ES014663 and ES020010, and Environmental Protection Agency (EPA) grant RD832416. The US Department of Veterans Affairs (VA) Normative Aging Study (NAS) is supported by the Cooperative Studies Program/ERIC, US Department of Veterans Affairs, and is a research component of the Massachusetts Veterans Epidemiology Research and Information Center (MAVERIC). Cognitive data collection at the VA NAS was supported by a VA Merit Review and a CSR\&D Research Career Scientist award to AS. The views expressed in this paper are those of the authors and do not necessarily represent the views of the US Department of Veterans Affairs. Additional support was provided by the US Department of Agriculture, Agricultural Research Service (contract 53-K06-510). MCP was supported by grants from the NIEHS (T32 ES007069) and National Institute of Aging (NIA) (F31 AG038233).

\section{Author details}

${ }^{1}$ Department of Environmental Health, Harvard School of Public Health, 665 Huntington Ave, Boston, MA 02115, USA. ²Department of Epidemiology, Harvard School of Public Health, 665 Huntington Ave, Boston, MA 02115, USA. ${ }^{3}$ INSERM U1052, Centre de Recherche en Cancérologie de Lyon, Lyon F-69000, France. ${ }^{4}$ Centre Léon Bérard, Pole de Recherche Translationnelle, Lyon F-69008, France. ${ }^{5}$ Department of Preventive Medicine, Northwestern University Feinberg School of Medicine, 420 East Superior St, Chicago, IL 60611, USA. ${ }^{6}$ VA Boston Healthcare System, Boston University Schools of Public Health and Medicine, 88E Newton St, Boston, MA 02118, USA.

Received: 12 February 2014 Accepted: 2 May 2014

Published: 30 May 2014 


\section{References}

1. Weuve J, Puett RC, Schwartz J, Yanosky JD, Laden F, Grodstein F: Exposure to particulate air pollution and cognitive decline in older women. Arch Intern Med 2012, 172:219-227.

2. Ranft U, Schikowski T, Sugiri D, Krutmann J, Kramer U: Long-term exposure to traffic-related particulate matter impairs cognitive function in the elderly. Environ Res 2009, 109:1004-1011.

3. Power MC, Weisskopf MG, Alexeeff SE, Coull BA, Spiro A 3rd, Schwartz J: Traffic-related air pollution and cognitive function in a cohort of older men. Environ Health Perspect 2011, 119:682-687.

4. Block ML, Calderon-Garciduenas L: Air pollution: mechanisms of neuroinflammation and CNS disease. Trends Neurosci 2009, 32:506-516.

5. DiMauro S, Schon EA: Mitochondrial respiratory-chain diseases. New England J Med 2003, 348:2656-2668.

6. Wallace DC, Brown MD, Melov S, Graham B, Lott M: Mitochondrial biology, degenerative diseases and aging. BioFactors (Oxford, England) 1998, 7:187-190.

7. Moulton PV, Yang W: Air pollution, oxidative stress, and Alzheimer's disease. J Environ Public Health 2012, 2012:472751.

8. Rego-Perez I, Fernandez-Moreno M, Fernandez-Lopez C, Arenas J, Blanco FJ: Mitochondrial DNA haplogroups: role in the prevalence and severity of knee osteoarthritis. Arthritis Rheum 2008, 58:2387-2396.

9. Torroni A, Achilli A, Macaulay V, Richards M, Bandelt HJ: Harvesting the fruit of the human mtDNA tree. Trends Genet 2006, 22:339-345.

10. Mancuso M, Filosto M, Orsucci D, Siciliano G: Mitochondrial DNA sequence variation and neurodegeneration. Hum Genomics 2008, 3:71-78.

11. Kruger J, Hinttala R, Majamaa K, Remes AM: Mitochondrial DNA haplogroups in early-onset Alzheimer's disease and frontotemporal lobar degeneration. Mol Neurodegener 2010, 5:8.

12. Bell $B$, Rose $C L$, Damon $A$ : The Veterans Administration longitudinal study of healthy aging. Gerontologist 1966, 6:179-184.

13. Spiro A, Vokonas P: The VA Normative Aging Study. In Encyclopedia of health and aging. Edited by Markides KS. CA: Thousand Oaks; 2007:421-422.

14. Tombaugh TN, Mclntyre NJ: The mini-mental state examination: a comprehensive review. J Am Geriatr Soc 1992, 40:922-935.

15. Weisskopf MG, Wright RO, Schwartz J, Spiro 3rd A, Sparrow D, Aro A, Hu H: Cumulative lead exposure and prospective change in cognition among elderly men: the VA Normative Aging Study. Am J Epidemiol 2004, 160:1184-1193.

16. Gryparis A, Coull BA, Schwartz J, Suh HH: Semiparametric latent variable regression models for spatiotemporal modelling of mobile source particles in the greater Boston area. J Roy Stat Soc C-App 2007, 56:183-209.

17. van Oven M, Kayser M: Updated comprehensive phylogenetic tree of global human mitochondrial DNA variation. Hum Mutat 2009, 30:E386-E394

18. Suissa S, Wang Z, Poole J, Wittkopp S, Feder J, Shutt TE, Wallace DC, Shadel GS, Mishmar D: Ancient mtDNA genetic variants modulate mtDNA transcription and replication. PLoS Genet 2009, 5:e1000474

19. Kazuno AA, Munakata K, Nagai T, Shimozono S, Tanaka M, Yoneda M, Kato $\mathrm{N}$, Miyawaki A, Kato T: Identification of mitochondrial DNA polymorphisms that alter mitochondrial matrix $\mathrm{pH}$ and intracellular calcium dynamics. PLoS Genet 2006, 2:e128.

20. Carugno M, Pesatori AC, Dioni L, Hoxha M, Bollati V, Albetti B, Byun HM, Bonzini M, Fustinoni S, Cocco P, Satta G, Zucca M, Merlo DF, Cipolla M, Bertazzi PA, Bacacrelli A: Increased mitochondrial DNA copy number in occupations associated with low-dose benzene exposure. Environ Health Perspect 2012, 120:210-215

21. Mo Y, Wan R, Feng L, Chien S, Tollerud DJ, Zhang Q: Combination effects of cigarette smoke extract and ambient ultrafine particles on endothelial cells. Toxicol In Vitro 2012, 26:295-303.

22. Li R, Ning Z, Cui J, Khalsa B, Ai L, Takabe W, Beebe T, Majumdar R, Sioutas C, Hsiai T: Ultrafine particles from diesel engines induce vascular oxidative stress via JNK activation. Free Radic Biol Med 2009, 46:775-782.

23. Naik E, Dixit VM: Mitochondrial reactive oxygen species drive proinflammatory cytokine production. J Exp Med 2011, 208:417-420

24. Brand MD: Uncoupling to survive? The role of mitochondrial inefficiency in ageing. Exp Gerontol 2000, 35:811-820.

25. Ruiz-Pesini E, Mishmar D, Brandon M, Procaccio V, Wallace DC: Effects of purifying and adaptive selection on regional variation in human mtDNA. science 2004, 303:223-226.
26. Wittkopp S, Staimer N, Tjoa T, Gillen D, Daher N, Shafer M, Schauer JJ, Sioutas C, Delfino RJ: Mitochondrial genetic background modifies the relationship between traffic-related air pollution exposure and systemic biomarkers of inflammation. PLoS One 2013, 8:e64444.

27. Autere J, Moilanen JS, Finnila S, Soininen H, Mannermaa A, Hartikainen P, Hallikainen M, Majamaa K: Mitochondrial DNA polymorphisms as risk factors for Parkinson's disease and Parkinson's disease dementia. Hum Genet 2004, 115:29-35.

28. Zeger SL, Thomas D, Dominici F, Samet JM, Schwartz J, Dockery D, Cohen A: Exposure measurement error in time-series studies of air pollution: concepts and consequences. Environ Health Perspect 2000, 108:419-426.

doi:10.1186/1476-069X-13-42

Cite this article as: Colicino et al:: Mitochondrial haplogroups modify the effect of black carbon on age-related cognitive impairment. Environmental Health 2014 13:42

\section{Submit your next manuscript to BioMed Central and take full advantage of:}

- Convenient online submission

- Thorough peer review

- No space constraints or color figure charges

- Immediate publication on acceptance

- Inclusion in PubMed, CAS, Scopus and Google Scholar

- Research which is freely available for redistribution

Submit your manuscript at www.biomedcentral.com/submit
C Biomed Central 